\title{
Primary hypothyroidism presenting as spontaneous ovarian hyperstimulation
}

\begin{abstract}
Spontaneous OHSS is an uncommon event occurring in absence of exogenous administration of gonadotropins. Most of the reported cases are associated with pregnancy. This report is of a case of primary hypothyroidism presenting only as spontaneous OHSS in a young unmarried girl. It needs to be remembered that at times this may also be an uncommon presentation of hypothyroidism.
\end{abstract}

Keywords: Hypothyroidism, Spontaneous OHSS, Thyroid stimulating hormone
Volume 7 Issue I - 2017

\section{Alka Goel}

Dr. Ram Manohar Lohia Hospital, India

Correspondence: Alka Goel, PGIMER \& DR RML HOPSITAL, New Delhi, India, Tel 97।7918884,

Email dralkagoel1963@gmail.com

Received: October 22, 2016 | Published: May 22, 2017

\section{Introduction}

Spontaneous ovarian hyperstimulation syndrome (spontaneous OHSS) is an uncommon event in the absence of exogenously administered gonadotropins. The condition was first reported by Van Wyk and Grumbach in $1960 .^{1,2}$ as a combination of polycystic ovaries, primary hypothyroidism and precocious puberty. From there on, there have been sporadic reports of OHSS, mostly in pre-pubertal or adolescent girls. ${ }^{3,4}$ The etiopathogenesis of spontaneous OHSS is yet to be completely understood.

\section{Case report}

A 17 year young, unmarried lady with no relevant medical history attended gynaecology out-patient department with pain abdomen for 6 months and first episode of delayed cycle. She attained menarche at 13 years with regular period ever since and denied any sexual activity. The patient was marginally short in comparison to siblings but had a normal development during infancy and childhood. Abdominal examination was normal. Investigations revealed moderate anaemia (haemoglobin $9.5 \mathrm{~g} / \mathrm{dl}$ ) and a normal serum biochemistry.

Ultrasound revealed small renal calculi in left kidneys with a normal pelvi-calyceal system. Uterus was normal with endometrial thickness of $6.89 \mathrm{~mm}$. Both ovaries were markedly enlarged (right $78.33 \mathrm{cc}$, left $124 \mathrm{cc}$ ), with multiple follicles $15-34 \mathrm{~mm}$ in size (Figure 1). Tumour markers (beta hCG, alpha-fetoprotein and Ca-125) were within normal limits. Thyroid functions revealed a high TSH (142.1) with high values of anti-thyroperoxidase (423.7). Other hormones (FSH, LH and Prolactin) were normal. Treatment was started with levo-thyroxine $50 \mu \mathrm{g}$, that was subsequently increased to $100 \mu \mathrm{g}$. The TSH values decreased to normal by 4 months, with normalization of menstrual periods. There was complete normalization of ovaries 2 months after achieving euthyroid state.

\section{Discussion}

OHSS is more commonly encountered as a complication of ovarian stimulation protocols. It can also be caused by FSH producing pituitary adenomas. Spontaneous OHSS has been mainly reported in cases with elevated levels of hCG as in pregnancy with or without hydatidiform mole..$^{5-8}$ or high levels of TSH as in hypothyroidism. There are very sporadic reports in non-pregnant unmarried ladies. ${ }^{9}$ One symptom commonly reported in all such cases was pain abdomen.

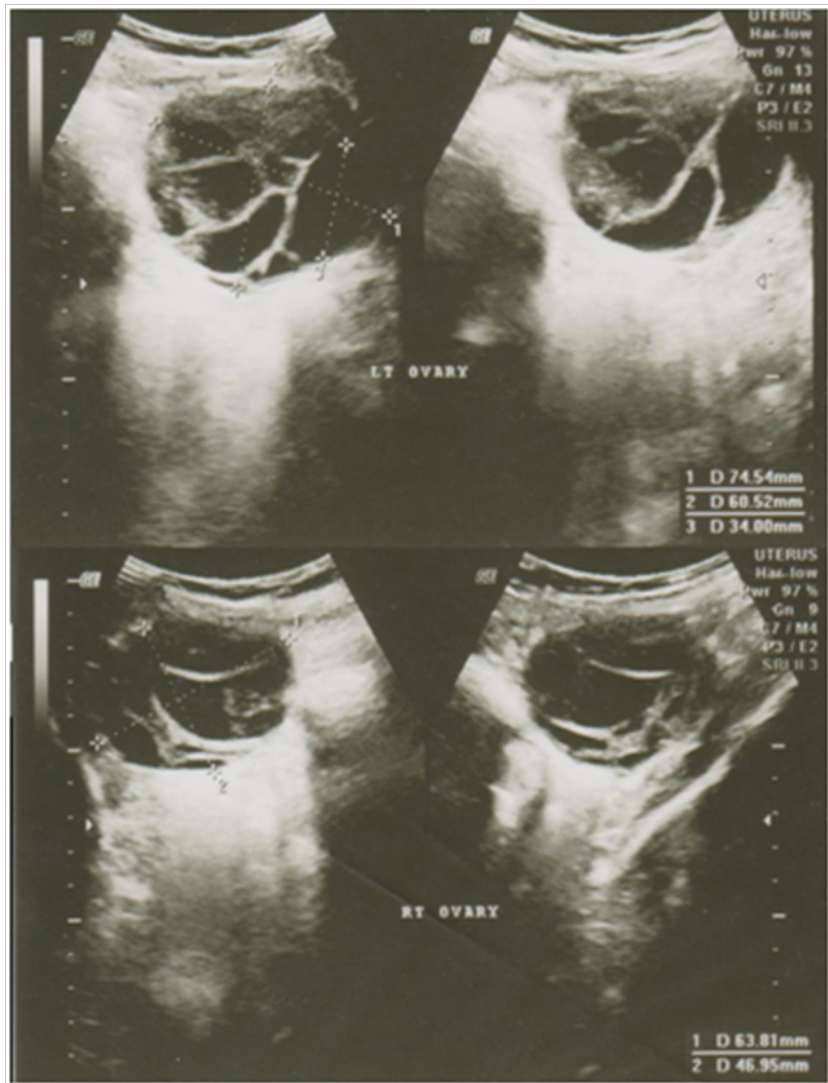

Figure I Ultrasound examination of Uterus.

OHSS has been classified into mild, moderate and severe, based on clinical presentation. ${ }^{10}$ Mild OHSS grade 1 presents with abdominal discomfort (presence of other abdominal symptoms such as nausea, vomiting and/or diarrhea make it grade 2) with ovarian enlargement of $5-12 \mathrm{~cm}$. Moderate OHSS (grade 3 ) has, in addition, sonographic evidence of ascites. It is only severe variety of OHSS, grade 4 and 5 , which presents with increased vascular permeability leading to shift of fluid into third space causing hemoconcentration, breathing difficulties, diminished renal perfusion and function and may even cause coagulation abnormalities. Majority of iatrogenic OHSS 
occurring during controlled ovarian hyperstimulation protocols develop mild OHSS. Likewise, all cases of spontaneous OHSS reported in literature presented with mild OHSS.

Both hCG and TSH have structural similarity to FSH. It has been postulated that mutation of FSHr gene can cause hyper responsiveness to circulating FSH or cross-responsiveness to hCG and TSH. De Leener et al. ${ }^{11}$ postulated three different mechanisms for Spontaneous OHSS. Type I, due to mutated FSH receptor, leads to recurrent OHSS. ${ }^{12}$ Type II, which is associated with high levels of hCG (as in hydatidiform mole and multiple gestation) is perhaps the most common. Type III, that is related to hypothyroidism. However, most cases described in literature are in pregnant patients with hypothyroidism, where there may be a synergistic action of high circulating levels of TSH and hCG.

However, two clear messages that emerge from the present case. First, is that spontaneous OHSS can also occur with primary hypothyroidism not associated with pregnancy. Secondly, OHSS may be the only presenting feature of hypothyroidism. The subject requires further study. It is possible that, if investigated, asymptomatic enlargement of ovaries with multiple follicles or OHS may exist in non-pregnant as well as pregnant ladies with hypothyroidism.

\section{Acknowledgments}

None.

\section{Conflicts of interest}

None.

\section{References}

1. Van Wyk JJ, Grumbach MM. Syndrome of precocious menstruation and galactorrhea in juvenile hypothyroidism: an example of hormonal overlap in pituitary feedback. J Pediatrics. 1960;57(3):416-435.
2. Jagadhish TK. Van Wyk and Grumbach syndrome (A syndrome of isosexual precocity and juvenile hypothyroidism). MJAFI. 2002;58(4):343-345.

3. Panico A, Lupoli GA, Fonderico F, et al. Multiple ovarian cysts in a young girl with severe hypothyroidism. Thyroid. 2007;17(12):2891293.

4. Gordon CM, Austin DJ, Radovick S, et al. Primary hypothyroidism presenting as severe vaginal bleeding in a prepubertal girl. J Pediatric Adolesc Gynecol. 1997;10(1):35-38.

5. Sridev S, Barathan S. Case report on spontaneous ovarian hyperstimulation syndrome following natural conception associated with primary hypothyroidism. J Hum Reprod Sci. 2013;6(2):158-161.

6. Langroudi RM, Amlashi FG, Emami MH. Ovarian cyst regression with levothyroxine in ovarian hyperstimulation syndrome associated with hypothyroidism. Endocrinol Diabetes Metab Case Rep. 2013;2013:130006.

7. Francisco C, Júlio C, Pinto G, et al. Ovarian hyperstimulation syndrome in a spontaneous pregnancy. Acta Med Port. 2011;24Suppl3:635-638.

8. Rachad M, Chaara H, Zahra Fdili F, et al. Ovarian hyperstimulation syndrome in a spontaneous pregnancy with invasive mole: report of a case. Pan Afr Med J. 2011;9:23.

9. Katulande P, Kariyawasam SS, Senanayake HM, et al. Multicystic ovaries and pituitary pseudo-adenoma associated with primary hypothyroidism. J Obstet Gynaecol. 2013;33(1):17-19.

10. Kumar P, Sait SF, Sharma A, et al. Ovarian Hyperstimulation Syndrome. J Human Reprod Sci. 2011;4(2):70-75.

11. DeLeener A, Caltabiano G, Erkan S, et al. Identification of the first germline mutation in the extracellular domain of the follitropin receptor responsible for spontaneous ovarian hyperstimulation syndrome. Hum Mutat. 2008;29(1):91-98.

12. Di Carlo C, Savoia F, Ferrara C, et al. Case report: a most peculiar family with spontaneous, recurrent ovarian hyperstimulation syndrome. Gynecol Endocrinol. 2012;28(8):649-651. 